\title{
Artroplastía Parcial de Cadera con Banda de Tensión en Fracturas de Fémur Proximal en Pacientes Ancianos
}

\author{
Partial Hip Arthroplasty with Tension Band in Proximal Femur Fractures in Older Patients
}

Gabriela Espín'; Washington Ruiz $^{1,3}$; Luis Espín V. ${ }^{3,4}$ \& Richard Silva ${ }^{2}$

ESPÍN, G.; RUIZ, W.; ESPÍN, V. L. \& SILVA, R. Artroplastía parcial de cadera con banda de tensión en fracturas de fémur proximal en pacientes ancianos. Int. J. Morphol., 37(1):363-368, 2019.

RESUMEN: Las fracturas intertrocantéricas en adulto mayor presentan gran dificultad para lograr una cicatrización ósea estable y una fijación en la artroplastía, para la recuperación completa y funcional del anciano. En pacientes adultos mayores sin stock óseo, se recomienda realizar anexo a la artroplastía de cadera, la colocación de una banda de tensión, con la finalidad de brindar mayor estabilidad a los fragmentos y sustentación a la artroplastía primaria, con el objetivo de lograr un retorno a la calidad de vida normal. A las cuatro semanas de la cirugía, se evidencia una mejora en la escala funcional alcanzando una puntuación media de 83, considerandose un buen resultado. En los controles subsecuentes se evidencia una media en la escala de Harris de 87. Los pacientes volvieron a su nivel de actividad preoperatoria, más de la mitad de los pacientes fueron capaces de caminar sin apoyos, y finalmente, no se reportaron complicaciones en los controles postquirúrgicos al seguimiento de un año.

PALABRAS CLAVE: Fracturas; Estabilización; Resultados.

\section{INTRODUCCIÓN}

Las fracturas intertrocantéricas son más frecuentes en pacientes ancianos y resultan en una alta morbilidad y una rehabilitación más dificultosa, producto del deterioro de la fuerza muscular y de la función propioceptiva (Puttakemparaju \& Beshaj, 2014).

Por estas razones, la cicatrización ósea estable es el elemento más crítico después de una fractura. Cuando se sugiere la artroplastía de cadera en el tratamiento de fracturas, la fijación estable del fragmento trocantérico mayor es esencial para la unión completa del hueso y la recuperación funcional de la articulación de la cadera (Thakur \& Lal, 2016).

Existen varias opciones quirúrgicas para el tratamiento de una fractura intertrocantérea de cadera inestable. La artroplastía es una alternativa de uso menos frecuente, pero permite al paciente llevar inmediatamente el peso completo. Muchas de las complicaciones de la fijación interna, incluyendo la migración del implante y la fractura no estabilizada, como la mala unión, se evitan mediante la realización de artroplastías (Navarro Collado et al., 2005).
La artroplastía aguda, en fracturas de fémur proximal, se encuentra recomendada en pacientes osteoporóticos y con artritis preexistente severa. Además, si se toma en cuenta que la fractura intertrocantérica inestable se presenta en el $45 \%$ a $52 \%$ de todas las fracturas de cadera de personas de edad avanzada, y que en la actualidad se encuentra recomendada la fijación interna y la artroplastía aguda, se debe evaluar adecuadamente el mejor tratamiento y manejo óptimo de estas fracturas femorales (Subramanian et al., 2012; Sun et al., 2017).

En pacientes adultos mayores sin stock óseo, se recomienda realizar, anexo a la artroplastía de cadera, la colocación de banda de tensión con la finalidad de brindar mayor estabilidad a los fragmentos y sustentación a la artroplastía primaria (Navarro Collado et al.; Sun et al.).

Con la asociación de una banda de tensión, con cerclaje a la artroplastía, la cual debe insertarse de forma endomedular, se garantiza una mejor estabilidad protésica y mejora la propiocepcion del paciente, beneficiándose el

\footnotetext{
${ }^{1}$ Médico Postgradista de Ortopedia y Traumatología, Residente $4^{\circ}$ año, Hospital Carlos Andrade Marín, Universidad San Francisco de Quito, Quito, Ecuador.

${ }^{2}$ Universidad Central del Ecuador, Quito, Ecuador.

${ }^{3}$ Hospital Carlos Andrade Marín, Quito, Ecuador.

${ }^{4}$ Doctorado en Ciencias Morfológicas, Universidad de La Frontera, Chile.
} 
postoperatorio para realizar ejercicios funcionales tempranos con cojinete de peso parcial (Sun et al.; Zhang et al., 2017).

El objetivo de este trabajo fue presentar la artroplastía parcial de cadera con banda de tensión como tratamiento de fracturas de fémur proximal en pacientes adultos mayores.

\section{MATERIAL Y MÉTODO}

Se desarrolló un estudio descriptivo comparativo de tratamiento en pacientes osteoporóticos adultos mayores del Hospital Carlos Andrade Marín, Quito, Ecuador. La muestra consistió en 12 pacientes, ingresados por emergencia con diagnóstico radiológico de fracturas intertrocantéricas complejas en el período del 1 de enero de 2016 al 31 diciembre de 2017. Se realizaron exámenes de laboratorio, radiológicos, radiografías anteroposteriores, laterales y axiales, y tomografías computadas.

Se utilizaron radiografías para la planificación preoperatoria adecuada (Fig. 1). Los pacientes fueron intervenidos quirúrgicamente bajo anestesia raquídea, en posición decúbito lateral, y se realizó un abordaje anterolateral (de Watson Jones) para exponer el fémur proximal, la cápsula y el acetábulo. Después de la incisión de la piel, se continuó con la fascia lateral del músculo, tensor de la facia lata y se separaron fibras del músculo glúteo máximo, a lo largo de la línea de incisión. La sección se extendió distalmente lo suficiente como para exponer la inserción tendinosa del músculo glúteo máximo (abordaje en promedio de $10 \mathrm{~cm}$ ). La cápsula articular se abrió utilizando una capsulotomía en forma de $\mathrm{T}$, colocándose un cerclaje para sostener el fragmento intertrocantérico, permitiendo realizar la luxación de cadera, evitando su desplazamiento. Posteriormente, se realizó una osteotomía subcapital para preservar el área del calcar, zona extremadamente sólida, correspondiente a la cortical inferior del cuello femoral que forma «el espolón o espina cervical inferior de Merkel, también denominado de Adams o simplemente calcar» (San Martín \& Velutini Kochen, 2011). Se evaluó la geometría de la fractura. Se extrajo y midió la cabeza femoral con la ayuda de pinzas de sujeción del hueso (Fig. 2). La preparación se realizó con escariadores y brocas.

Con respecto a la banda de tensión, después de la preparación del canal femoral, se realizó una perforación sobre la cara anterolateral del fémur proximal perforando solo la cortical a unos 4 a $5 \mathrm{~cm}$ distales al sitio de la fractura. Se utilizaron $30 \mathrm{~cm}$ de alambre de cerclaje, el cual se dobló por la mitad, y se pasaron dos alambres de acero

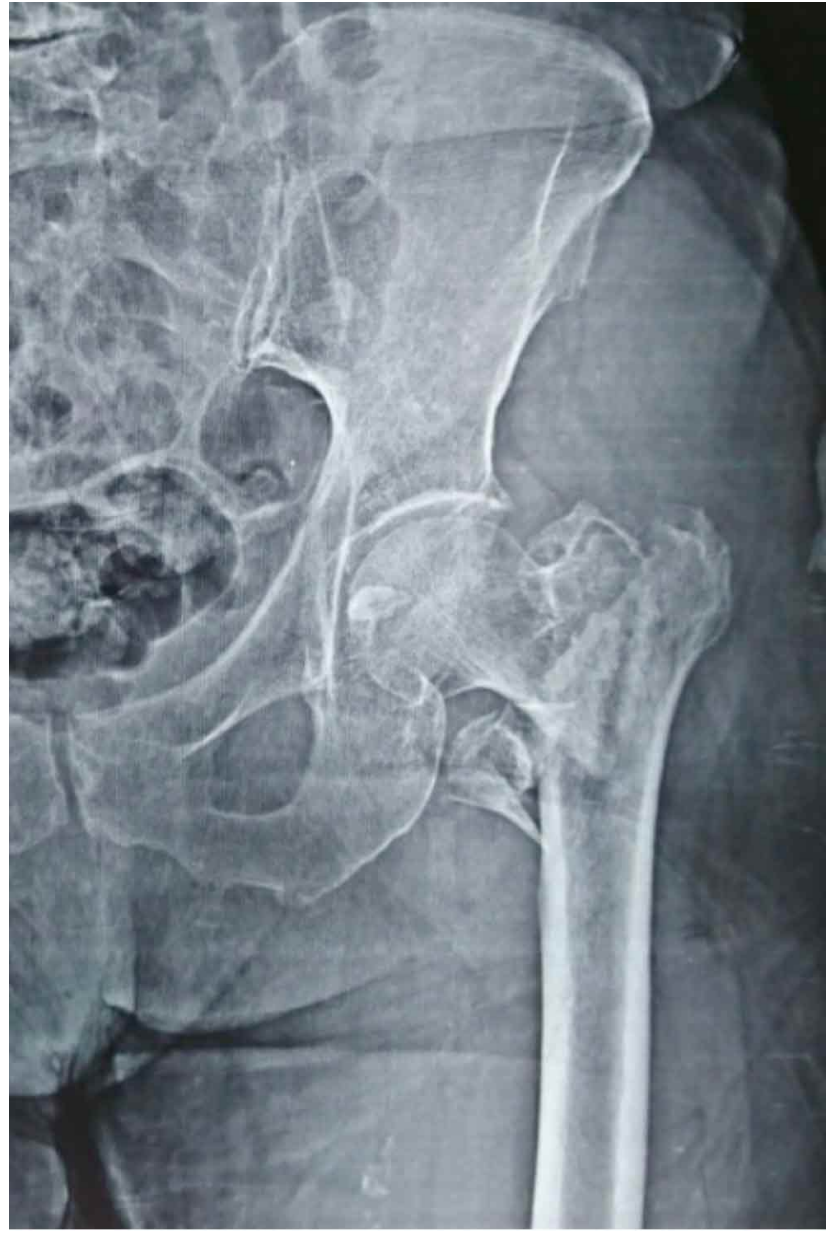

Fig. 1. Paciente de 85 años con fractura intertrocantérica AO $31 \mathrm{~A}$ 2 , Tronzo IIIB.

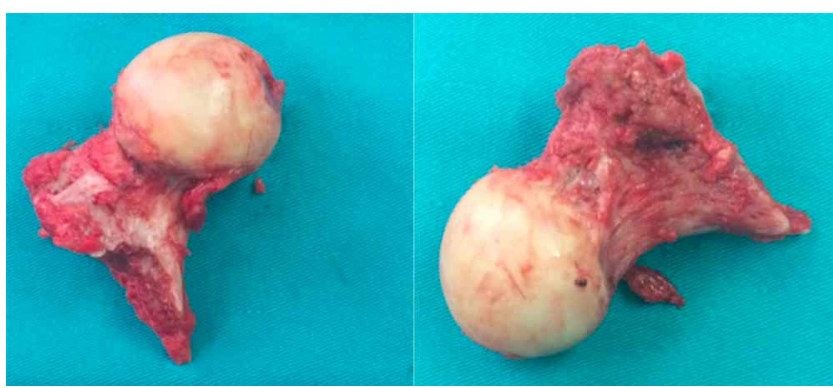

Fig. 2. Fragmento óseo de la cabeza femoral, extraída por fractura intertrocantérica.

inoxidable a través de este forámenes hacia el interior del canal femoral, saliendo a través del forámen consecutivo y el otro extremo sobre la cara anterolateral del fémur proximal (Figs. 3 y 4 ).

El tallo del vástago de ensayo se insertó teniendo cuidado de la anteversión, que fue juzgada por el eje largo de la pierna para alcanzar la misma longitud del miembro, 


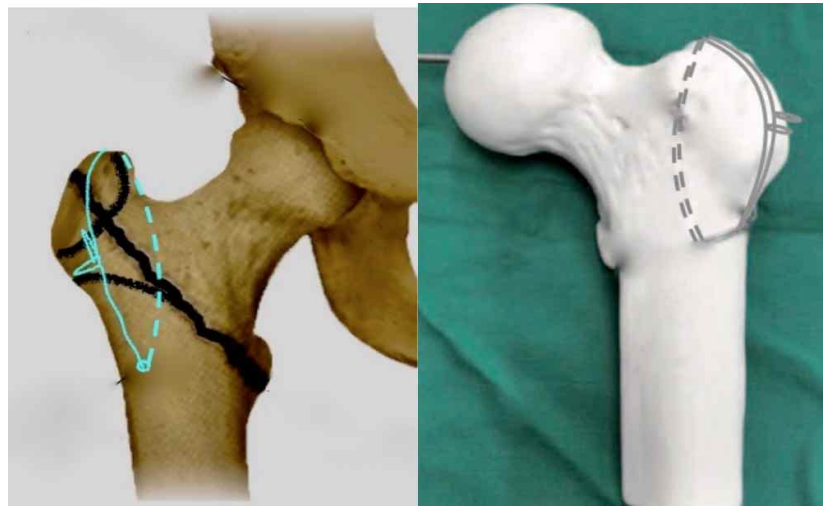

Fig. 3. Diseño de banda de tensión endomedular, con doble alambre.

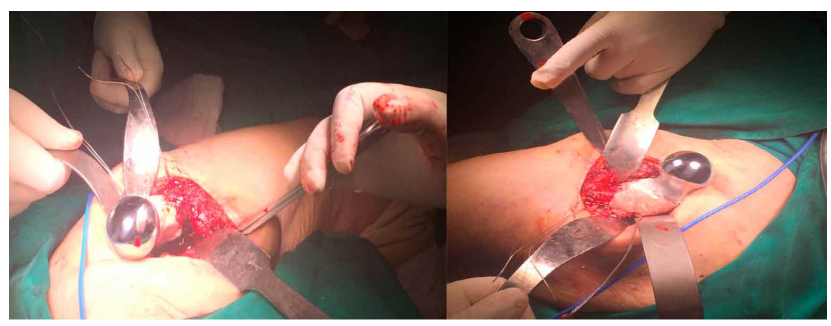

Fig. 4. Reducción de prótesis parcial con banda de tensión.

que también fue comprobada por la prueba de Shuck con el desplazamiento de no más de 2 - $3 \mathrm{~mm}$. Se realizó la reducción del ensayo y se evaluó la estabilidad de la cadera.

Se utilizó cemento de baja viscosidad para lograr una mejor penetración a través de los forámenes perforados.

Antes de insertar el implante final, se realizó la cementación y se reconstruyó el calcar. Después de la reducción de la cadera con el implante final, los principales fragmentos de fractura del trocánter mayor se aproximaron entre sí con la ayuda de los dos alambres de cerclaje que se pasaron superior al trocánter mayor, dentro de la inserción del músculo glúteo medio, de medial a lateral sobre el músculo.

Para finalizar la cirugía, el resto del tejido blando se cerró en capas. Se utilizaron cefalosporinas de segunda generación de un solo disparo preoperatoriamente $(2 \mathrm{~g}$ de cefazolina previa a la incisión en la inducción, con la administración de 4 dosis después de la cirugía, 1 g cada 6 h).

Con el fin de prevenir la trombosis venosa profunda (TVP), se utilizaron heparina fraccionada y medias elásticas antiembólicas bilaterales. A los pacientes se les permitió la deambulación con carga completa en el primer día postoperatorio.

\section{RESULTADOS}

Los pacientes fueron evaluados sobre la base de su autoevaluación con respecto al bienestar físico, social, mental y alivio en el dolor. Se modificó la puntuación de cadera de Harris para estandarizar los resultados (Figs. 5, 6 y 7).

La inspección primaria de la herida se realizó el segundo día postoperatorio. Los pacientes comenzaron a sentarse después de comprobar las radiografías y al día siguiente se permitió al paciente caminar con la ayuda de un andador. Los pacientes fueron dados de alta el $4^{\circ}$ día postoperatorio y seguidos a las 2 semanas para revisión primaria de herida. Posteriormente, fueron evaluados a las 4 semanas, 10 semanas, 24 semanas, y un año de la cirugía.

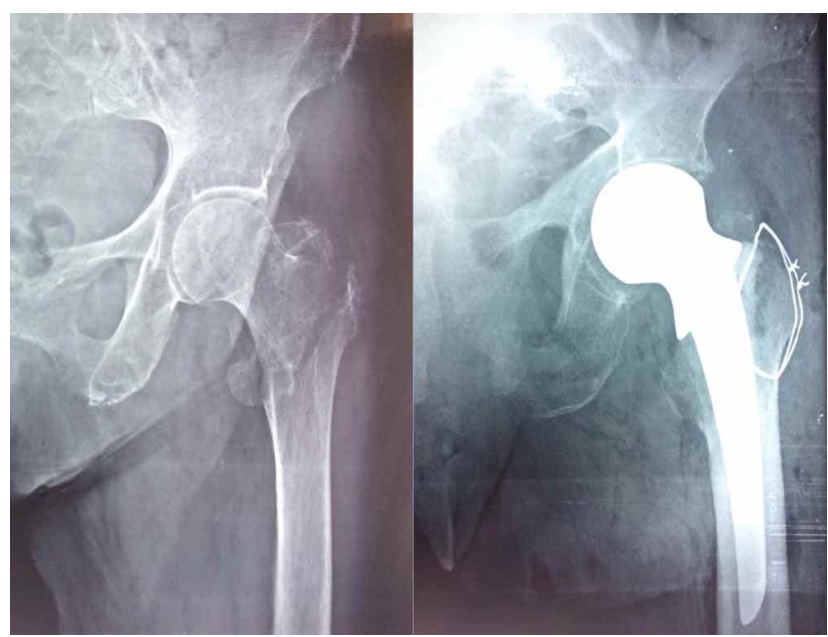

Fig. 5. Fractura intertrocantérica Tronzo IV, en paciente femenina de 85 años, resuelta con prótesis parcial tipo Thompson cementado, con banda de tensión.

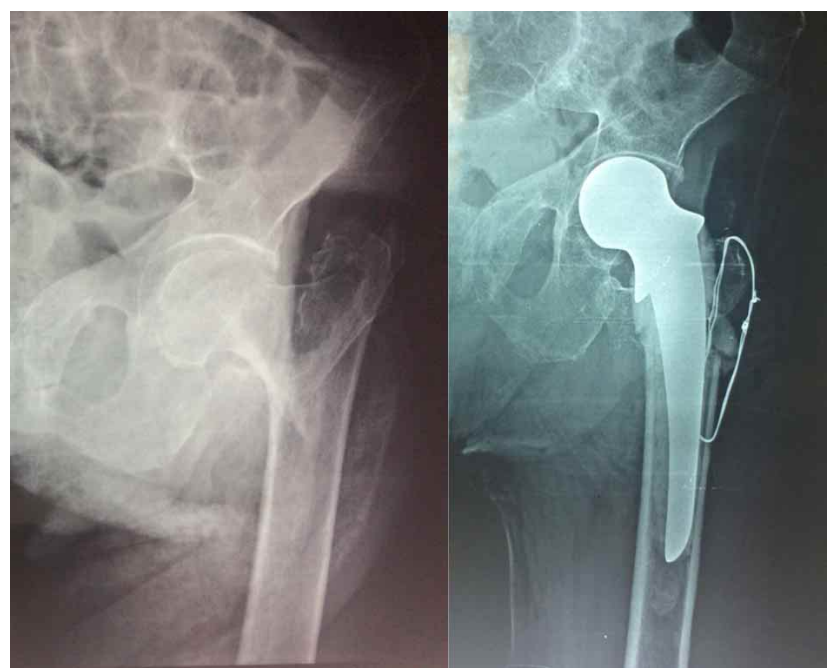

Fig. 6. Fractura intertrocantérica Tronzo IV, en paciente femenina de 87 años, resuelta con prótesis parcial tipo Thompson cementado, con banda de tensión. 


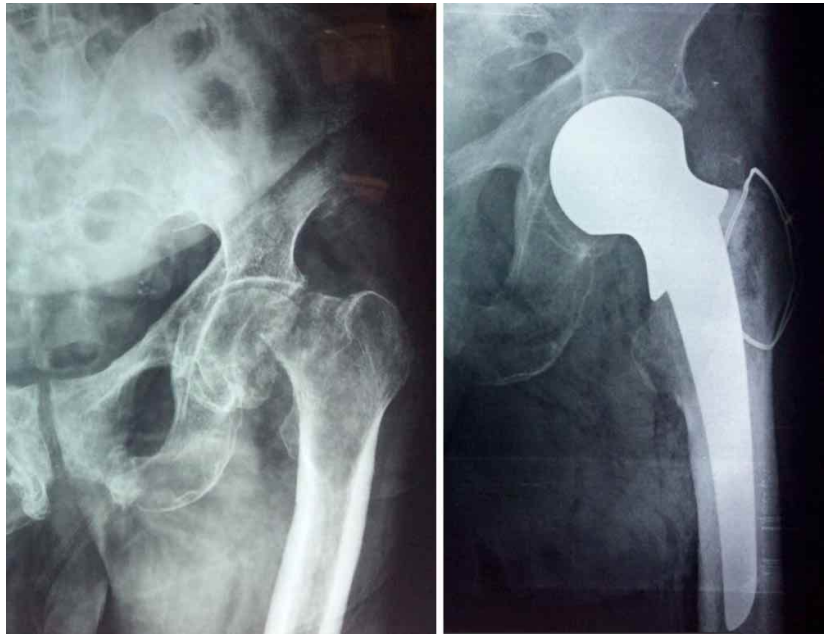

Fig. 7. Fractura subcapital de fémur, en paciente femenina de 82 años, resuelta con prótesis parcial tipo Thompson cementado, con banda de tensión.

En el primer control postquirúrgico, los pacientes fueron sometidos a la escala de Harris, presentando una puntuación media de 73 (resultado aceptable). En el segundo control, realizado a las 4 semanas postquirúrgicas, se evidenció una mejora en la escala funcional teniendo como puntuación media 83 (buen resultado). En los controles subsecuentes, se evidenció una media en la escala de Harris de 87.

La mayoría de los pacientes volvieron a su nivel de actividad preoperatoria; más de la mitad de los pacientes fueron capaces de caminar sin apoyos. En cada visita se realizó una evaluación radiológica, sin reportarse complicaciones en los controles postquirúrgicos al seguimiento de 1 año de los pacientes.

\section{DISCUSIÓN}

La hemiartroplastía se ha utilizado para fracturas intertrocantéricas inestables desde 1971. El reemplazo protésico primario en pacientes con fractura intertrocantérica y osteoporóticos inestables no está asociado con todas estas complicaciones aunque es una cirugía técnicamente exigente, asociándose con menores tasas de complicaciones. Además, si a este método se le anexa la técnica de banda de tensión, la estabilidad protésica y la propiocepción del paciente le brinda una restauración temprana de motricidad, con mejores resultados en la rehabilitación (Lee et al., 2011).

Hay suficiente evidencia en la literatura para apoyar la hemiartroplastía con banda de tensión como opción primaria de tratamiento en estos tipos de fracturas. Debido a que permite realizar carga temprana y no hay riesgo de co- lapso en varo del fragmento de fractura, la rehabilitación es rápida y las complicaciones como escaras, infección torácica y atelectasia son notablemente inferiores. Permite un retorno precoz al nivel de actividad previo a la fractura y evita esencialmente el agravamiento de las enfermedades comórbidas (Sancheti et al., 2010; Siwach et al., 2012).

Stern \& Goldstein (1977) utilizaron la prótesis de Leinbach para el tratamiento primario de 22 fracturas pertrocantéreas tipo AO / OTA 31A2.3 y encontraron una ambulación temprana y un retorno precoz al estado de prefractura como ventaja definitiva. Han recomendado el reemplazo protésico para permitir la carga inicial de peso en pacientes adultos mayores con una fractura intertrocantérea inestable. Por su parte, Choi et al. (2010) han demostrado resultados favorables en el seguimiento a corto plazo después de realizar artroplastía de cadera para fracturas intertrocantéricas inestables (Sidhu et al., 2010; Ukaj et al., 2017).

Un estudio prospectivo aleatorizado que comparó los tornillos de compresión de cadera de la hemiprótesis de Vandeputte, utilizados en estas fracturas, sugirió que el reemplazo protésico primario podría tener menos complicaciones precoces y resultados funcionales satisfactorios. Otros estudios también han demostrado una mayor tasa de complicaciones en los pacientes tratados con osteosíntesis, en comparación con la artroplastía, lo que lleva a una mayor aplicación de técnicas de artroplastía, garantizando la estabilidad protésica si se le asocia la banda de tensión (Kim et al., 2005; Geiger et al., 2007).

En un estudio que aplican la hemiartroplastía primaria para el tratamiento de fracturas femorales pertrocantéricas inestables (AO/OTA tipo 31 A2.3), en pacientes osteoporóticos mayores, se demuestra que la artroplastía es un procedimiento seguro y eficaz, mostrando una capacidad anterior para soportar el peso total del cuerpo, con una tasa de recuperación más rápida y una mejor calidad de vida (Waddell et al., 2004; Zhang et al.).

En un estudio que analizaron el método de fijación según el tipo de fractura del trocánter mayor en fracturas intertrocantéricas inestables sometidas a artroplastía, se concluyó que los diferentes métodos de fijación aplicados a las fracturas con fijación o artroplastía fueron exitosos, logrando la reducción y fijación adecuadas de las grandes fracturas trocantéricas. También se observaron mejores resultados de unión ósea cuando se realizó artroplastía con banda de tensión en pacientes con fracturas intertrocantéricas inestables (Berend et al., 2004; Frisch et al., 2015).

En fracturas intertrocantéricas inestables con el fragmento trocantérico mayor, la falta de unión del fragmento 
trocantérico mayor puede causar dolor en la región trocantérica, anomalía funcional de la marcha y dislocación debido a la reducción de la fuerza de los "músculos abductores de la cadera", como son considerados los músculos glúteos medio y menor. Por lo tanto, la reducción anatómica y la fijación rígida del trocánter mayor son importantes. Las técnicas o dispositivos de fijación se deben realizar previamente con alambre, para realizar una banda de tensión trocantérica; otro método anexo puede ser la aplicación de una placa de garra (Navarro Collado et al.).

Nam et al. (2017) han reportado resultados satisfactorios con artroplastía total de cadera sin cemento y cableado de banda de doble tensión en la fijación de fracturas intertrocantéricas inestables con el mayor fragmento trocantérico. Además, Choi et al. documentaron resultados satisfactorios con hemiartroplastía de cadera y cableado de cerclaje en la fijación de fracturas intertrocantéreas inestables (Frisch et al.). Grimsrud et al. (2005) realizaron un estudio en 39 pacientes con fracturas intertrocantéricas inestables tratadas con hemiartroplastía cementada, sumada a banda de tensión, y comprobaron que este tratamiento fue un método eficaz sin complicaciones, comparable a otros estudios (Sidhu et al.).

Kim et al. han recomendado desarrollar hemiartroplastía cementada como una opción útil para el tratamiento de fracturas intertrocantéreas inestables en pacientes mayores de 65 años y se han obtenido buenos resultados en el $88 \%$ de los pacientes (Geiger et al.).

En la fractura triturada de los trocánteres mayor y menor, los cirujanos pueden enfrentar retos técnicos durante la cirugía, en casos de fijación de hilos no rígida o deformidad ósea en direcciones no deseadas a pesar de la ligera tensión del hilo. Dado que el trocánter mayor sirve como el principal sitio de unión de los "músculos abductores", la reducción anatómica y el mantenimiento de la reducción dependiendo de las posiciones de los miembros inferiores son inquietantes en los casos de trituración del trocánter mayor (Haidukewych \& Berry, 2003).

Hamadouche et al. (2001) han logrado resultados satisfactorios con el uso de banda de tensión, para obtener mayor unión trocantérica tras la artroplastía de cadera. En estas fracturas presentan una escala funcional buena, pero la tasa de dislocación es más alta en comparación con la hemiartroplastía en individuos ancianos. La fijación del trocánter mayor a la construcción restante, con ayuda de hilos de acero inoxidable y suturas no absorbibles, tiene que ser meticulosa para evitar la sacudida en el período postoperatorio temprano y para mejorar la propiocepcion (Berend et al.; Frisch et al.).
En estudios publicados por Navarro Collado et al. y Siwach et al., reportaron una puntuación media en la escala de Harris de 86,4. La mayoría de los pacientes volvieron a su nivel de actividad preoperatoria; el $64 \%$ de los pacientes fueron capaces de caminar sin apoyo preoperatorio; el $40 \%$ de los pacientes fueron capaces de caminar sin soporte en el seguimiento final; mientras que el $36 \%$ fueron dependientes de apoyos (andador) después de la cirugía.

\section{CONCLUSIONES}

La elección del método apropiado en pacientes adultos mayores, con mala calidad ósea y patrones de fractura distintos, puede ayudar a adquirir una mejor reducción y fijación apropiadas de fracturas trocantéricas mayores.

La hemiartoplastía es un tratamiento eficaz para las fracturas de fémur proximal desplazadas en los pacientes mayores de 65 años. Se ha encontrado que tiene una menor tasa de complicaciones y una menor tasa de reoperación.

La reconstrucción de fragmentos de hueso femoral metafisario y la readhesión trocantérica alrededor de un vástago del componente femoral, implican técnicas que han logrado consistentemente resultados satisfactorios. Se restablece la masa ósea proximal del fémur y se consigue una elevada tasa de cicatrización trocantérica. La hemiartroplastía de cadera disminuye las complicaciones, como migración de material, y se puede realizar la carga total temprana.

Con el fin de mejorar tanto la estabilidad protésica, como el método de unión trocantérico, se recomienda la banda de tensión como un elemento efectivo utilizado en conjunto con las artroplastías, lo que probablemente coayudaría en la deambulación temprana y la rehabilitación, permitiendo a la vez, el regreso temprano a las actividades de la vida diaria, mejorando la propiocepcion y reduciendo la morbimortalidad.

En este trabajo aporta con una nueva técnica de realización de banda de tensión en hemiartroplastía, la cual reduce el tiempo quirúrgico, requiere una menor incisión y además, evita el apoyo en las partes blandas del brazo de palanca, estando el apoyo en el macizo trocantérico, convirtiendo las fuerzas de distracción en compresión.

La evaluación funcional, según la escala de Harris, demostró una mejora en los resultados de la técnica de hemiartroplastía con banda de tensión. 
ESPÍN, G.; RUIZ, W.; ESPÍN, V. L. \& SILVA, R. Partial hip arthroplasty with tension band in proximal Femur fractures in older patients . Int. J. Morphol., 37(1):363-368, 2019.

SUMMARY: The intertrochanteric fractures in the elderly are very difficult to achieve stable bone healing and fixation in arthroplasty for complete and functional recovery of the elderly. In elderly patients without bone stock, it is recommended to make an attachment to the hip arthroplasty the placement of tension band with the purpose of providing greater stability to the fragments and support to the primary arthroplasty with the aim of a return to the normal quality of life, in the results at 4 weeks post-surgery an improvement in the functional scale is evidenced having as average score 83 taken as good results. In the subsequent controls a mean on the Harris scale of 87 was evidenced, the patients returned to their level of preoperative activity, more than half of the patients were able to walk without support, no complications were reported in the postoperative follow-up controls. of 1 year of patients.

KEY WORDS: Fractures; Stabilization; Results.

\section{REFERENCIAS BIBLIOGRÁFICAS}

Berend, K. R.; Lombardi, A. V. Jr.; Mallory, T. H.; Chonko, D. J.; Dodds, K. L. \& Adams, J. B. Cerclage wires or cables for the management of intraoperative fracture associated with a cementless, tapered femoral prosthesis: results at 2 to 16 years. J. Arthroplasty, 19(7 Suppl. 2):1721, 2004.

Choi, JK; Gardner, TR; Yoon, E .; Morrison, TA; Macaulay, WB \& Geller, JA El efecto de la técnica de fijación en la rigidez de las fracturas periprotésicas de FD de Vancouver B1. J. Arthroplast., 25 (6 Suppl.): 124, 2010.

Frisch, N. B.; Charters, M. A.; Sikora-Klak, J.; Banglmaier, R. F.; Oravec, D. J. \& Silverton, C. D. Intraoperative Periprosthetic Femur Fracture: A Biomechanical Analysis of Cerclage Fixation, J. Arthroplasty, 30(8):1449-57, 2015.

Geiger, F.; Zimmermann-Stenzel, M.; Heisel, C.; Lehner, B. \& Daecke, W. Trochanteric fractures in the elderly: the influence of primary hip arthroplasty on 1-year mortality. Arch. Orthop. Trauma Surg., 127(10):959-66, 2007.

Grimsrud, C.; Monzon, R. J.; Richman, J. \& Ries, M. D. Cemented hip arthroplasty with a novel cerclage cable technique for unstable intertrochanteric hip fractures. J. Arthroplasty, 20(3):337-43, 2005.

Haidukewych, G. J. \& Berry, D. J. Hip arthroplasty for salvage of failed treatment of intertrochanteric hip fractures. J. Bone Joint Surg. Am., 85-A(5):899-904, 2003.

Hamadouche, M .; Kerboull, L .; Meunier, A .; Courpied, JP \& Kerboull, M. Artroplastia total de cadera para el tratamiento de caderas anquilosadas: un estudio de seguimiento de cinco a veintiún años. $J$. Bone Joint Surg. Am., 83-A (7):992-8, 2001.

Kim, S. Y.; Kim, Y. G. \& Hwang, J. K. Cementless calcar-replacement hemiarthroplasty compared with intramedullary fixation of unstable intertrochanteric fractures. A prospective, randomized study. J. Bone Joint Surg. Am., 87(10):2186-92, 2005.

Lee, Y. K.; Ha, Y. C.; Chang, B. K.; Kim, K. C.; Kim, T. Y. \& Koo, K. H. Cementless bipolar hemiarthroplasty using a hydroxyapatite-coated long stem for osteoporotic unstable intertrochanteric fractures. J. Arthroplasty, 26(4):626-32, 2011.

Nam, D .; Riegler, V .; Clohisy, JC; Nunley, RM \& Barrack, RL El impacto de la artroplastia total de cadera en el movimiento pélvico y la posi- ción del componente funcional es muy variable. J. Arthroplasty., 32 (4):1200-5, 2017.

Navarro Collado, M. J.; Peiró Moreno, S.; Ruiz Jareño, L.; Payá Rubio, A.; Hervás Juan, M. T. \& López Matéu, P. Validez de la escala de cadera de Harris en la rehabilitación tras artroplastía de cadera. Rehabilitación, 39(4):145-94, 2005.

Puttakemparaju, K. V. \& Beshaj, N. R. Unstable intertrochanteric fracture in elderly treated with bipolar hemiarthroplasty: A prospective case series. Afr. J. Trauma, 3(2):81-6, 2014.

San Martín, R. A. \& Velutini Kochen, J. A. Anatomy of the human femoral head. orthopedic issues, part II. Biomechanics and microscopic morphology. Int. J. Morphol., 29(2):371-6, 2011.

Sancheti, Kh.; Sancheti, P.; Shyam, A.; Patil, S.; Dhariwal, Q. \& Joshi, R. Primary hemiarthroplasty for unstable osteoporotic intertrochanteric fractures in the elderly: A retrospective case series. Indian J. Orthop., 44(4):428-34, 2010

Sidhu, A. S.; Singh, A. P.; Singh, A. P. \& Singh, S. Total hip replacement as primary treatment of unstable intertrochanteric fractures in elderly patients. Int. Orthop., 34(6):789-92, 2010.

Siwach, R.; Jain, H.; Singh, R. \& Sangwan, K. Role of hemiarthroplasty in intertrochanteric fractures in elderly osteoporotic patients: a case series. Eur. J. Orthop. Surg. Traumatol., 22:467-72, 2012.

Stern, MB \& Goldstein, TB El uso de la prótesis de Leinbach en las fracturas intertrocánticas de la cadera. Clinica Ortopedia Relat. Res., 128:325-31, 1977.

Subramanian, G. V.; Guravareddy, A. V.; Reddy, A. K. \& Chiranjeevi, T. Greater Trochanter Reconstruction in Unstabl Intertrochanteric Fractures Treated With Cemented Bipolar Hemiarthroplasty: A Technical Note. J. Orthop. Case Rep., 2(3):28-30, 2012.

Sun, D.; Park, B. S.; Jang, G. I. \& Lee, B. The fixation method according to the fracture type of the greater trochanter in unstable intertrochanteric fractures undergoing arthroplasty. Hip Pelvis, 29(1):62-7, 2017.

Thakur, A. \& Lal, M. Cemented hemiarthroplasty in elderly osteoporotic unstable trochanteric fractures using fracture window. Malays. Orthop. J., 10(1):5-10, 2016.

Ukaj, S.; Gjyshinca, B.; Podvorica, V.; Ukaj, F.; Molliqaj, G.; Boshnjaku, A. \& Gamulin, A. Primary hemiarthroplasty for treatment of unstable pertrochanteric femoral fractures (AO/OTA Type 31 A2.3) in elderly osteoporotic patients. SICOT J., 3:31, 2017.

Waddell, J. P.; Morton, J. \& Schemitsch, E. H. The role of total hip replacement in intertrochanteric fractures of the femur. Clin. Orthop. Relat. Res., (429):49-53, 2004.

Zhang, H.; Xu, Z.; Zhou, A.; Yan, W.; Zhao, P.; Huang, X. \& Zhang, J. Efficacy of Kirschner-wires and tension band in hip arthroplasty for aged patients with unstable intertrochanteric osteoporotic fracture: A 2-to-11-year follow-up. Medicine (Baltimore), 96(1):e5614, 2017.

Dirección para Correspondencia:

Luis Espín Villamarin

Hospital Pediátrico Baca Ortiz.

Av. Colon y 6 de Diciembre

Quito

ECUADOR

Email: luisesp10@hotmail.com

Recibido: $16-09-2018$

Aceptado: $20-11-2018$ 